\title{
Modelling interfacial properties with spot-DGT-ePC-SAFT for binary mixtures including ionic liquid-based systems
}

\author{
Yunhao Sun ${ }^{\mathrm{a}, \mathrm{b},}$, Xiaohua Lu ${ }^{\mathrm{b}}$, Xiaoyan $\mathrm{Ji}^{\mathrm{a},{ }^{*}}$ \\ ${ }^{\mathrm{a} D i v i s i o n}$ of Energy Science/Energy Engineering, Luleå University of Technology, 97187 Luleå, \\ Sweden \\ bState Key Laboratory of Materials-Oriented Chemical Engineering, Nanjing Tech University, \\ Nanjing 210009, P. R. China
}

\footnotetext{
Corresponding Authors

*E-mail: yunhao.sun@ltu.se.

*E-mail: xiaoyan.ji@1tu.se.
} 
Table S1. Full names of IL-ions studied in this work.

\begin{tabular}{cc}
\hline IL-ion & Full name \\
\hline$\left[\mathrm{C}_{\mathrm{n}} \mathrm{mim}\right]^{+}$ & 1-n-alkyl-3-methylimidazolium cation \\
{$\left[\mathrm{C}_{\mathrm{n}} \mathrm{py}\right]^{+}$} & 1-n-alkyl-pyridinium cation \\
{$\left[\mathrm{Tf}_{2} \mathrm{~N}\right]^{-}$} & Bis(trifluoromethyl-sulfonyl)amide anion \\
{$\left[\mathrm{PF}_{6}\right]^{-}$} & Hexafluorophosphate anion \\
{$\left[\mathrm{BF}_{4}\right]^{-}$} & Tetrafluoroborate anion \\
{$\left[\mathrm{C}_{2} \mathrm{SO}_{4}\right]^{-}$} & Ethylsulfate anion \\
\hline
\end{tabular}

